\title{
Simultaneous synthesis and separation of nanoparticles using aqueous two phase systems
}

\author{
Krishna V Kinhal ${ }^{1}$, Shashwata Sinha ${ }^{1}$, Aishwarya Ravisankar ${ }^{1}$, Nirav P Bhatt ${ }^{2}$, Subramaniam \\ Pushpavanam ${ }^{1}$ \\ ${ }^{1}$ Department of Chemical Engineering, ${ }^{2}$ Department of Biotechnology, \\ Indian Institute of Technology Madras, Chennai-600036, India.
}

\section{SUPPORTING INFORMATION}

\section{S1. Determination of Binodal curve and tie-line length:}

To determine the binodal curve, stock solutions for PEG (40 wt \%) and citrate (30 wt \%) were prepared. All required solutions were prepared using deionized water. All weighing was carried out using an analytical weighing balance (BT 224S, Sartorius AG, Germany) with a precision of $\pm 0.001 \mathrm{~g}$. Experiments were carried out at constant temperature $\left(30^{\circ} \mathrm{C}\right)$ and at different $\mathrm{pH}$ $(4,7$, and 11) to test the effect of $\mathrm{NaOH}$ on the binodal curve. The $\mathrm{pH}$ values of the stock solutions were adjusted individually to the required value, by adding an appropriate ratio of $\mathrm{NaOH}(1 \mathrm{M})$ and $\mathrm{H}_{2} \mathrm{SO}_{4}(98 \%)$ to the solutions. The values were measured with a $\mathrm{pH}$ meter (LI 617 , ELICO, India) with a precision of \pm 0.01 .

The binodal curves were determined experimentally using the cloud-point method. ${ }^{1}$ For this, the concentrated (30 wt \%) stock solution of salt (citrate) was added drop-wise to a known amount $(5 \mathrm{~g})$ of the concentrated $(40 \mathrm{wt} \%)$ stock solution of PEG 6000 in a conical flask with continuous stirring until turbidity appeared. Turbidity was used as an indication of the twophase formation. A small volume of the solution ( $2 \mathrm{ml})$ was centrifuged at $13000 \mathrm{rpm}$ for 10 minutes to confirm two-phase formation. The overall composition of the system at the point of two phase formation was noted. On confirming the cloud-point, the biphasic system was diluted drop-wise with water until turbidity disappeared and the solution became clear. This indicated the transition from two-phase to single-phase region by addition of water. The salt solution was again added drop-wise until the system entered the two-phase region. The binodal composition was determined by calculating the amount of PEG and citrate and water added in the above experiments.

To determine the tie line of the binodal curve, the refractive index was used for calibration (Figure S1) to determine the concentration of PEG in both the phases. The conductivity was measured and calibrated (Figure S2) at pH 11 to estimate the concentration of citrate in both the phases. It was assumed that the citrate does not influence the refractive index of the solution, and PEG does not affect the conductivity of the solution. 


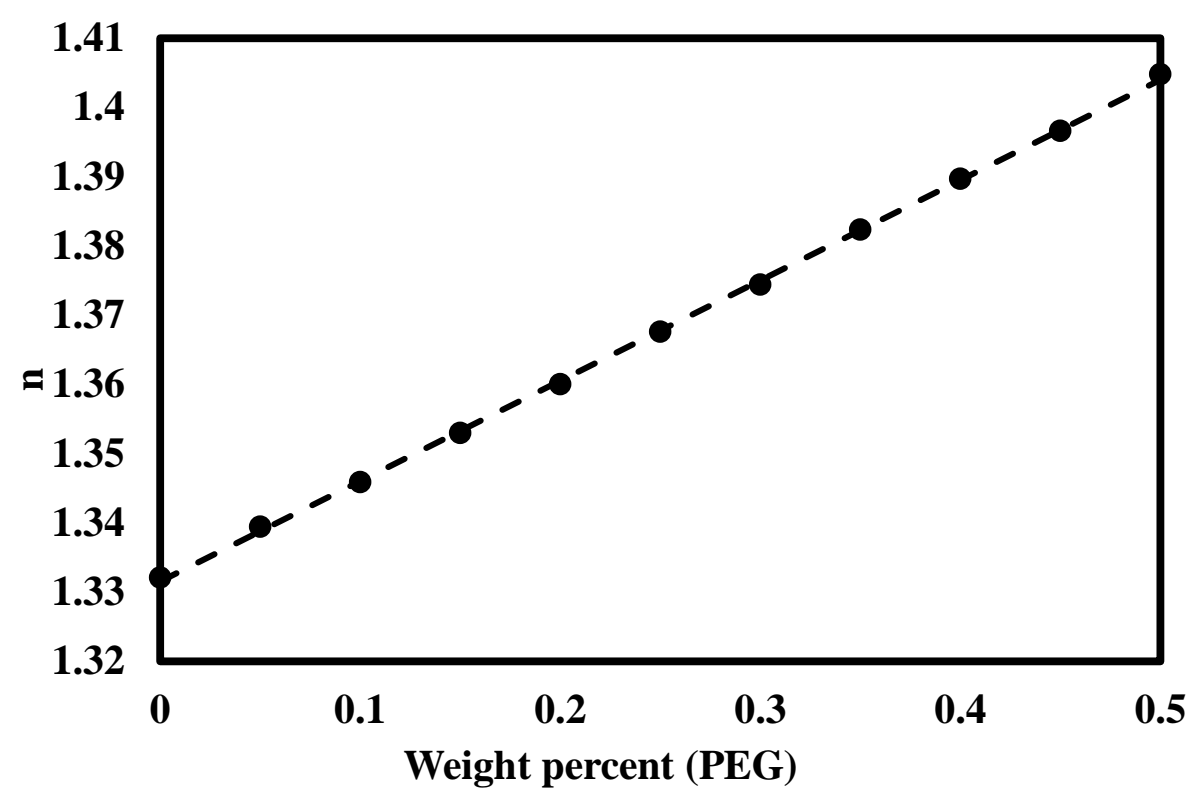

Figure S1. Refractive Index calibration curve for determination of PEG concentration

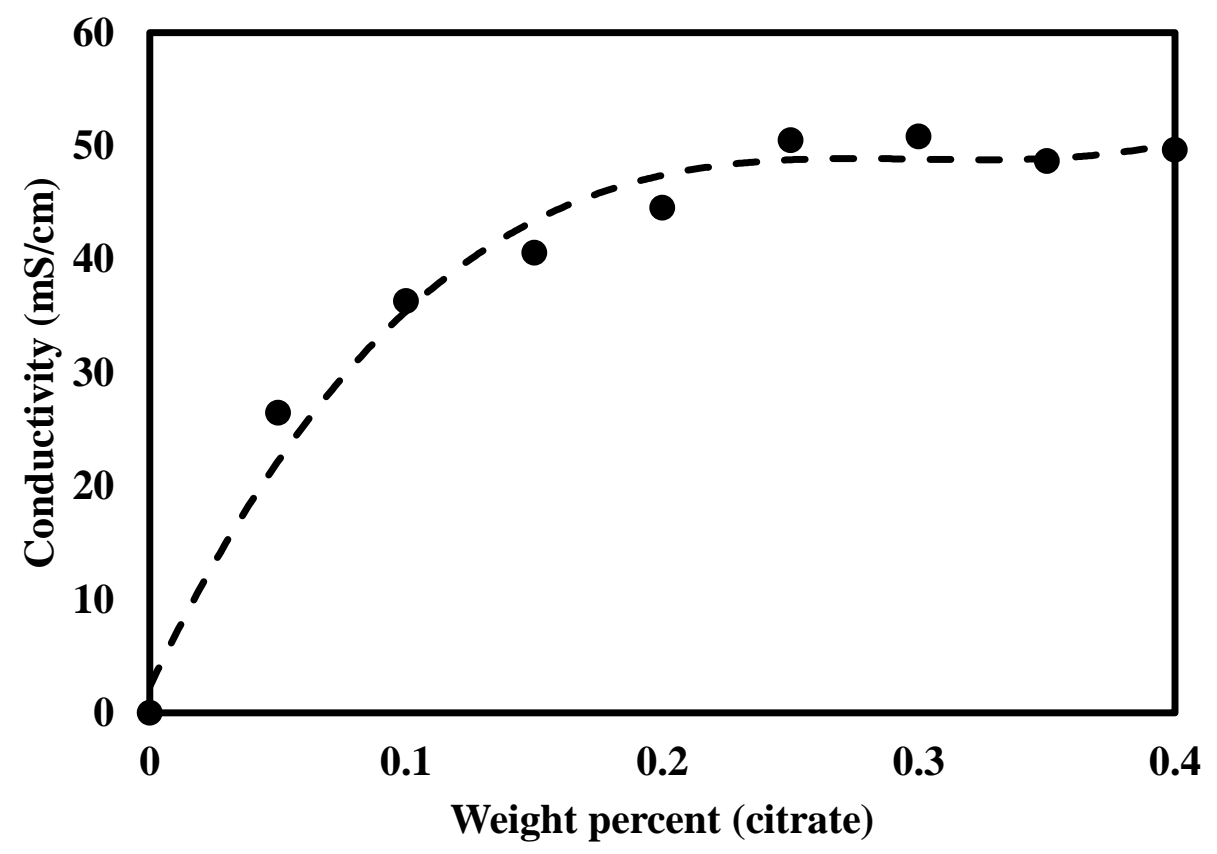

Figure S2. Conductivity calibration curve for determining the concentration of citrate in the solution.

\section{S5.1 Mass Balance:}

A composition of $12 \mathrm{wt} \%$ citrate and $12 \mathrm{wt} \%$ PEG yielded $66 \mathrm{ml}$ of citrate rich phase and 54 $\mathrm{ml}$ of PEG rich phase. To verify the mass balance, the mass of citrate and PEG in each phase (citrate rich and PEG rich) was estimated using tie-line data. The estimated mass was compared with the actual amount of PEG and citrate added to the system. These results summarized in Table S1 indicate an accuracy of around 5\% in our measurements.

\section{i) Citrate component balance}

Amount of citrate added for $12 \mathrm{wt} \%$ composition $=15.789 \mathrm{gms}$ 
Total mass of citrate estimated at the equilibrium of ATPS = mass of citrate in the citrate-rich phase + mass of citrate in the PEG-rich phase

$=\left(\left(\frac{18.09}{100}\right) * 66 * 1.11586\right)+\left(\left(\frac{5.34}{100}\right) * 54 * 1.10488\right)$

$=16.508 \mathrm{gms}$

\section{ii) PEG component balance:}

Amount of PEG added for $12 \mathrm{wt} \%$ composition $=15.789 \mathrm{gms}$

Total mass of PEG estimated at the equilibrium of ATPS = mass of PEG in the citrate-rich phase + mass of PEG in the PEG-rich phase

$$
\begin{aligned}
& =\left(\left(\frac{0.87}{100}\right) * 66 * 1.11586\right)+\left(\left(\frac{24.18}{100}\right) * 54 * 1.10488\right) \\
& =15.067 \mathrm{gms}
\end{aligned}
$$

\begin{tabular}{|l|l|l|l|l|l|}
\hline Component & \multicolumn{1}{|c|}{$\begin{array}{c}\text { mass in } \\
\text { PEG rich } \\
(\mathbf{g m s})\end{array}$} & $\begin{array}{c}\text { mass in } \\
\text { citrate rich } \\
(\mathbf{g m s})\end{array}$ & $\begin{array}{c}\text { Total Mass } \\
\text { estimated } \\
(\mathbf{g m s})\end{array}$ & $\begin{array}{c}\text { Total mass } \\
\text { added (gms) }\end{array}$ & $\begin{array}{c}\text { Percent } \\
\text { error }\end{array}$ \\
\hline Citrate & 3.186 & 13.322 & 16.508 & 15.789 & +4.57 \\
\hline PEG & 14.426 & 0.641 & 15.067 & 15.789 & -4.55 \\
\hline
\end{tabular}

Table S1. Mass balance of each component in two phases of ATPS

\section{S2. Size estimation of nanoparticles:}

For size estimation, a total of 100-150 particles in the TEM image were considered, and the Ferret's diameter was measured for each particle using ImageJ software provided by the National Institute of Health, USA. TEM images of silver nanoparticles obtained in pure citrate, pure PEG, PEG rich and, citrate rich phases are shown in Figures S3, S4, S5, S6, respectively. The images are acquired at different locations of the TEM grid.

a)

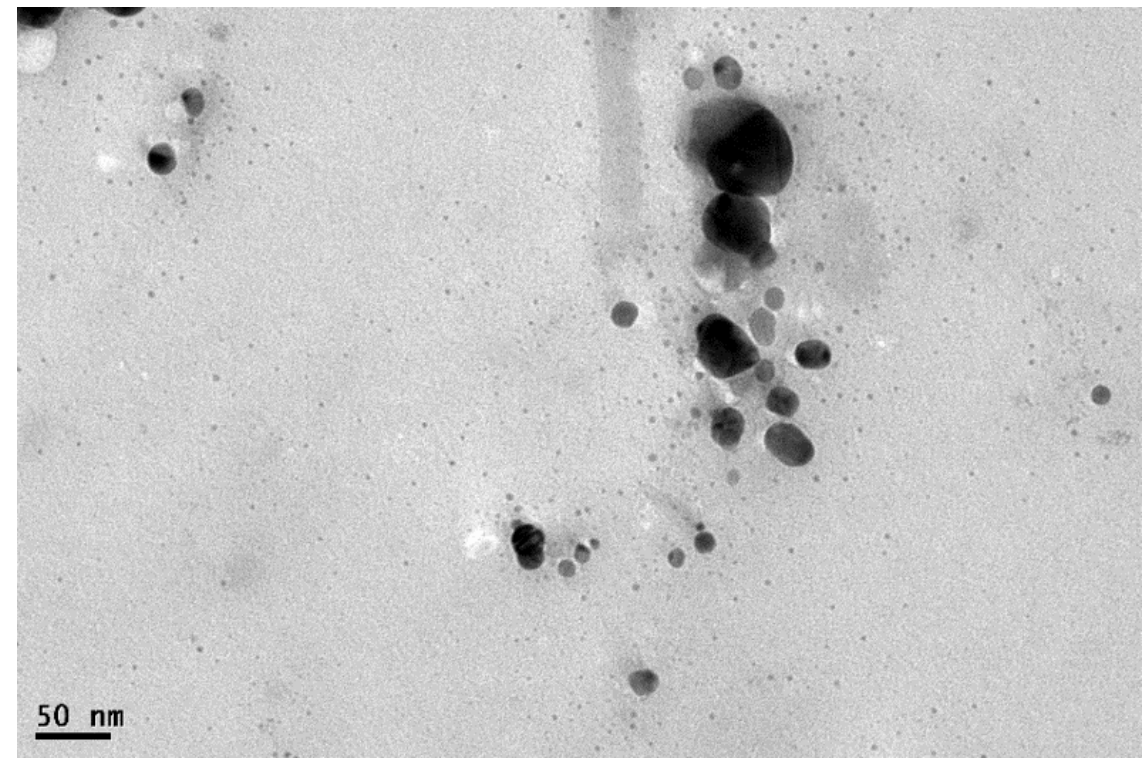


b)

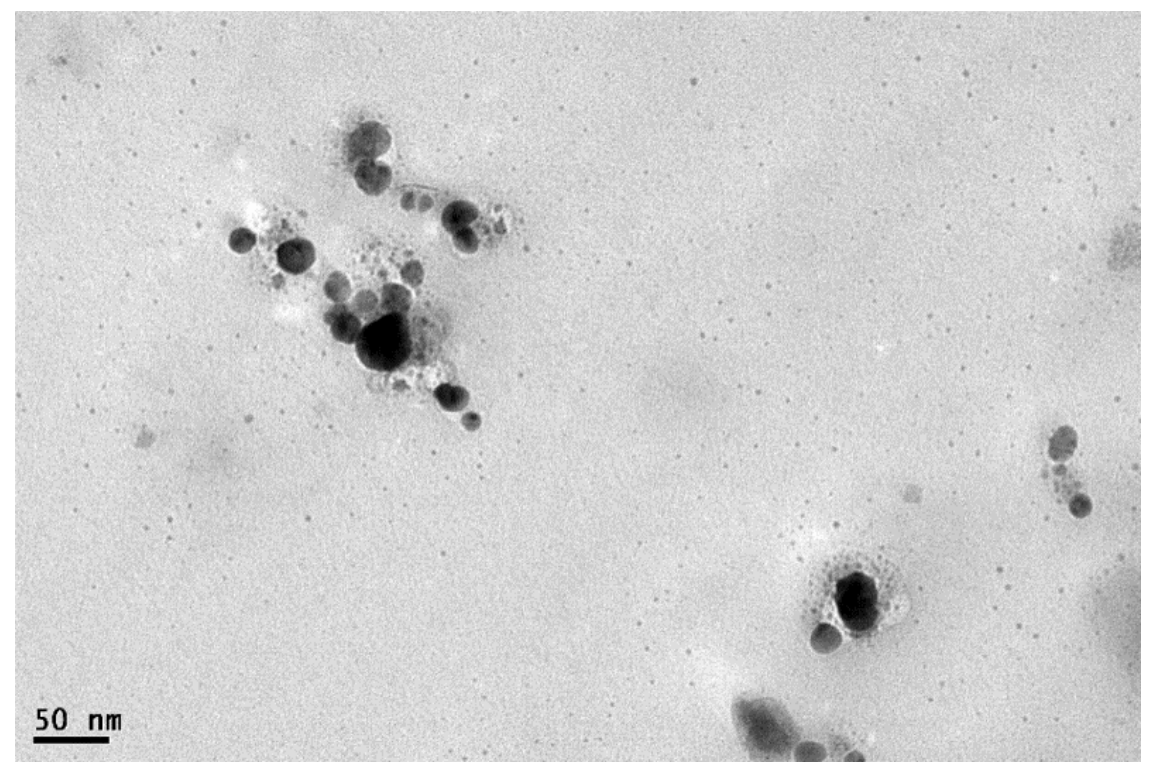

c)

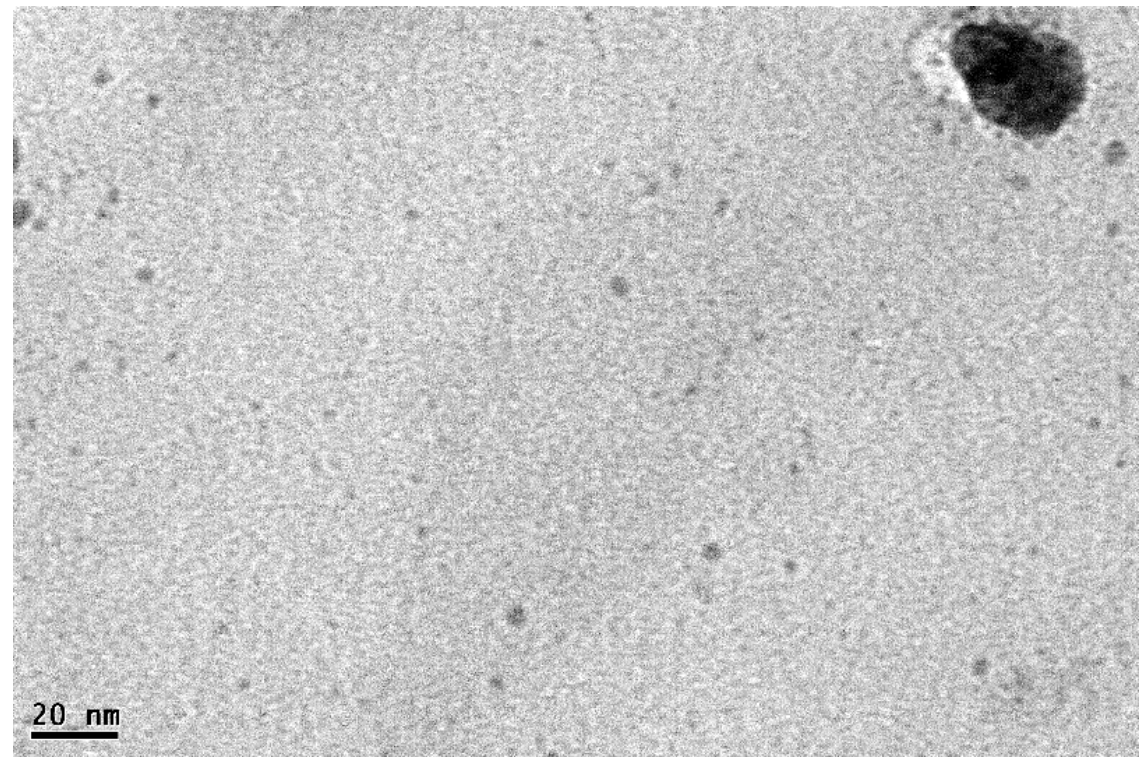

Figure S3. a) b) c) TEM images of silver nanoparticle synthesized in pure citrate phase acquired at different locations in the TEM grid 
a)

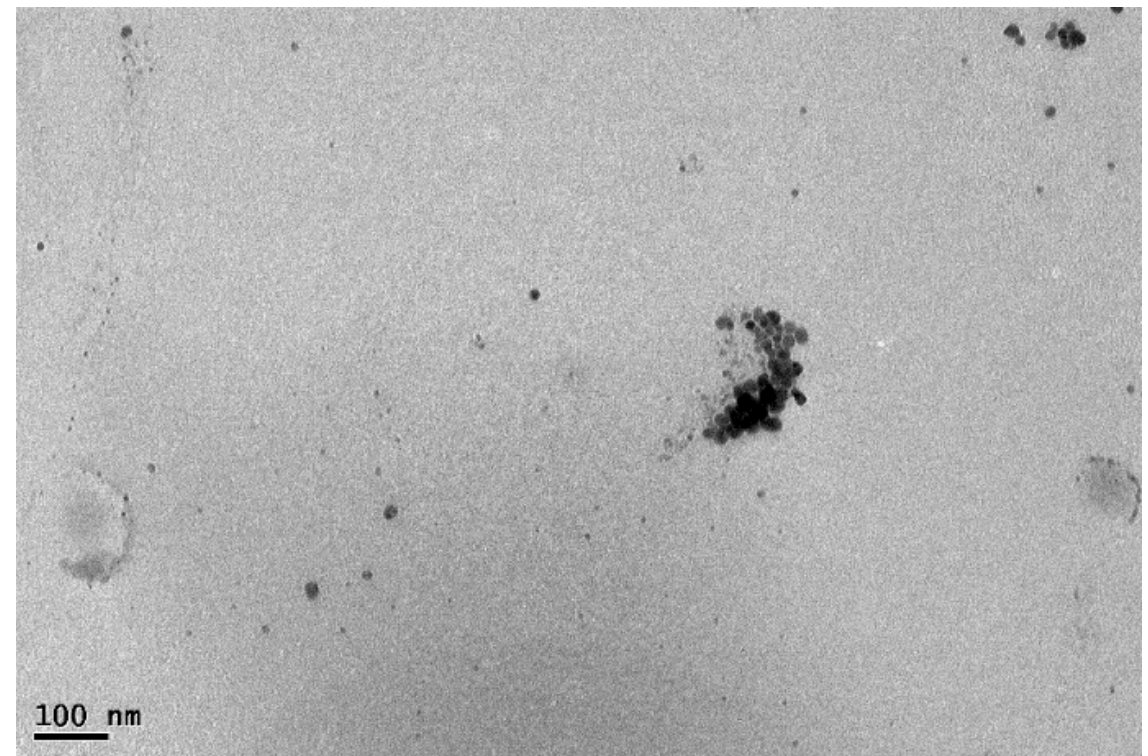

b)

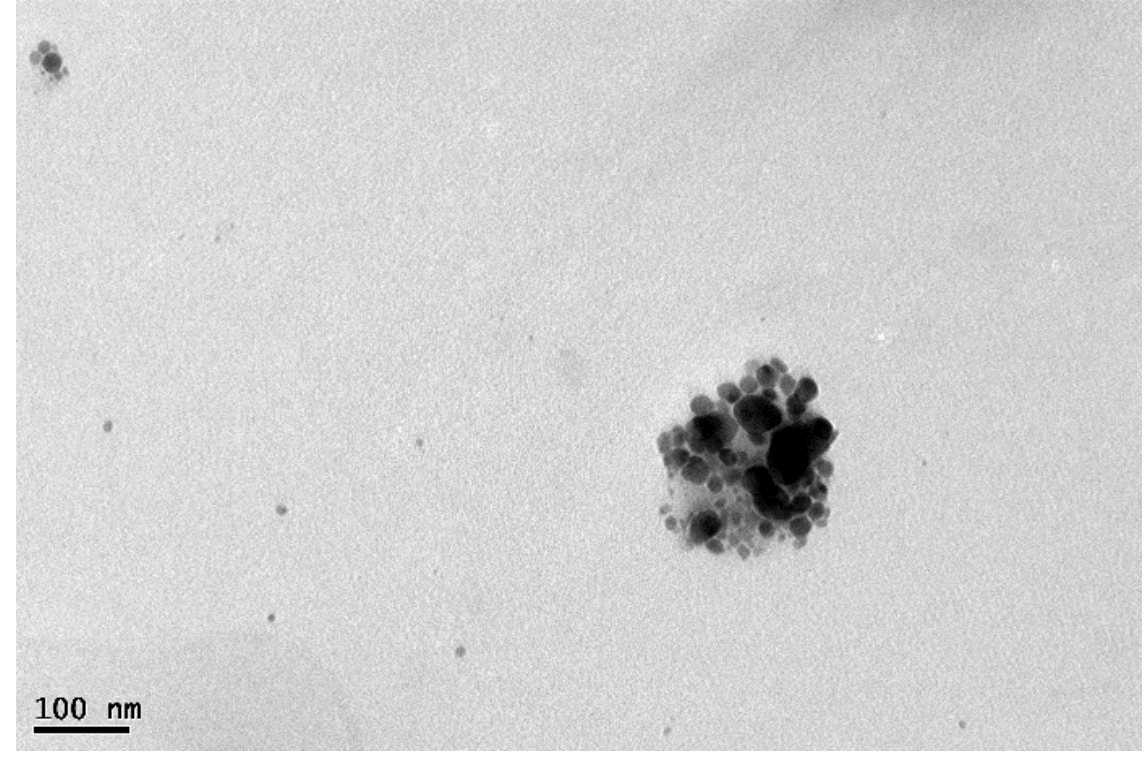

c)

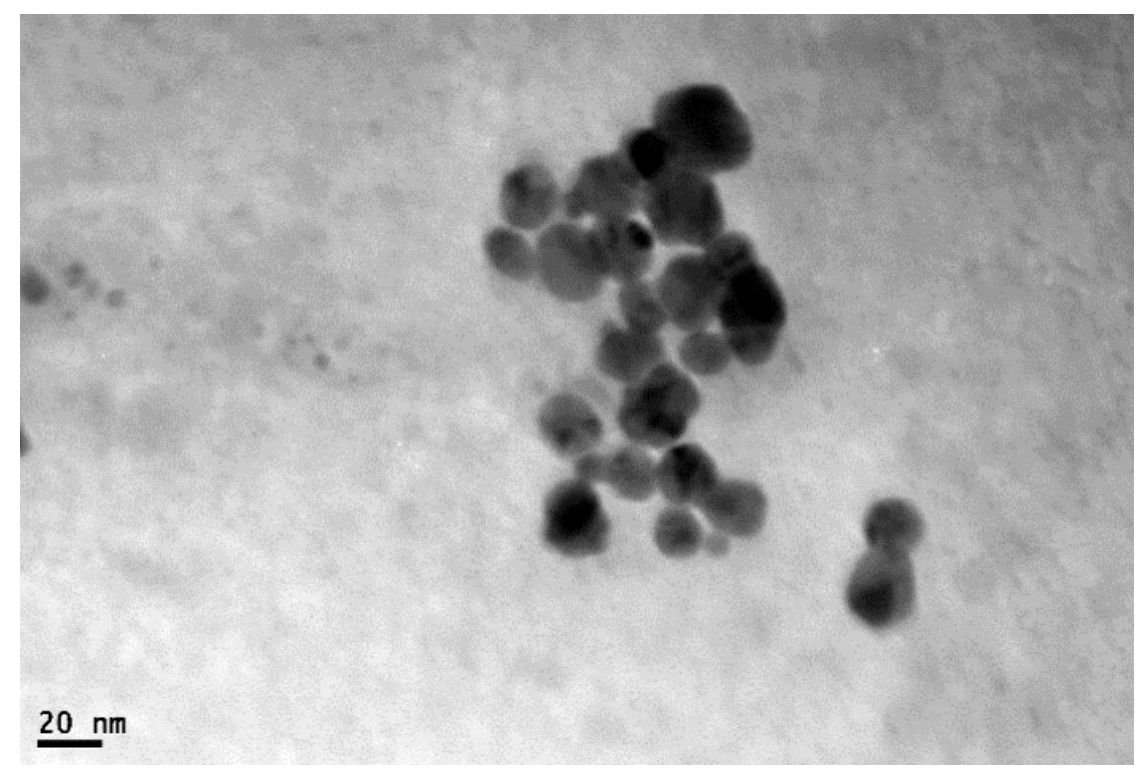

Figure S4. a) b) c), images of silver nanoparticle synthesized in pure PEG phase acquired at different locations of the TEM grid 
a)

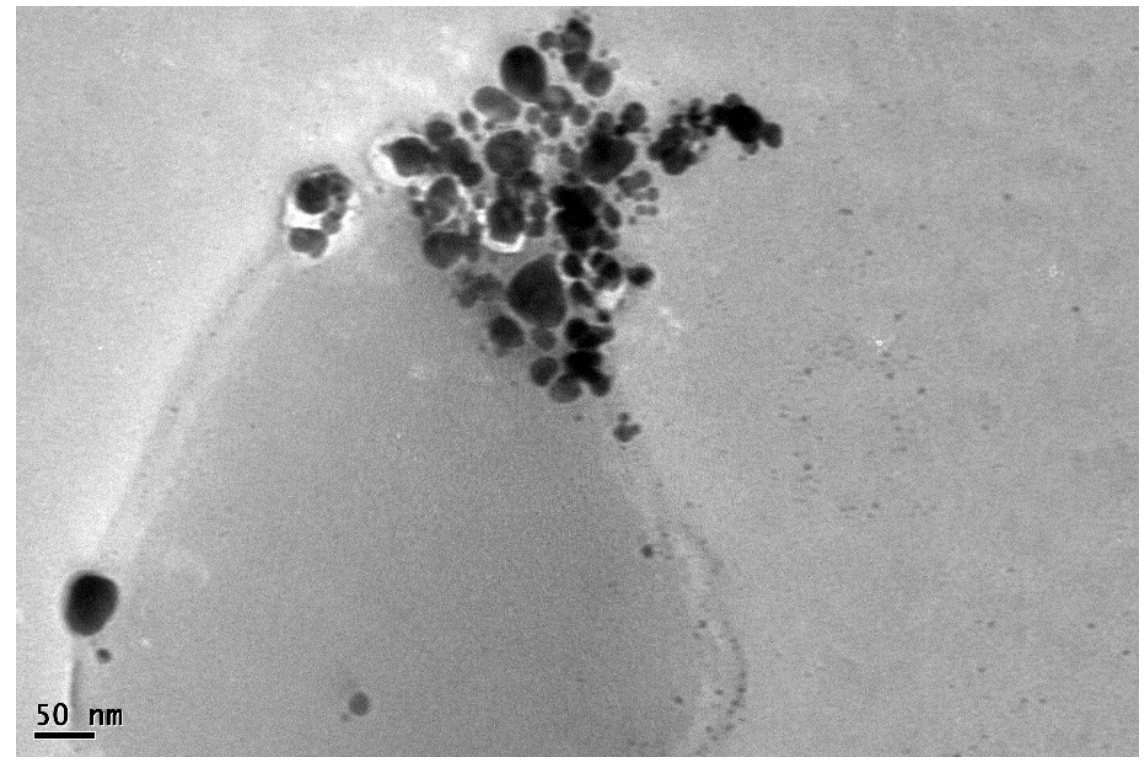

b)

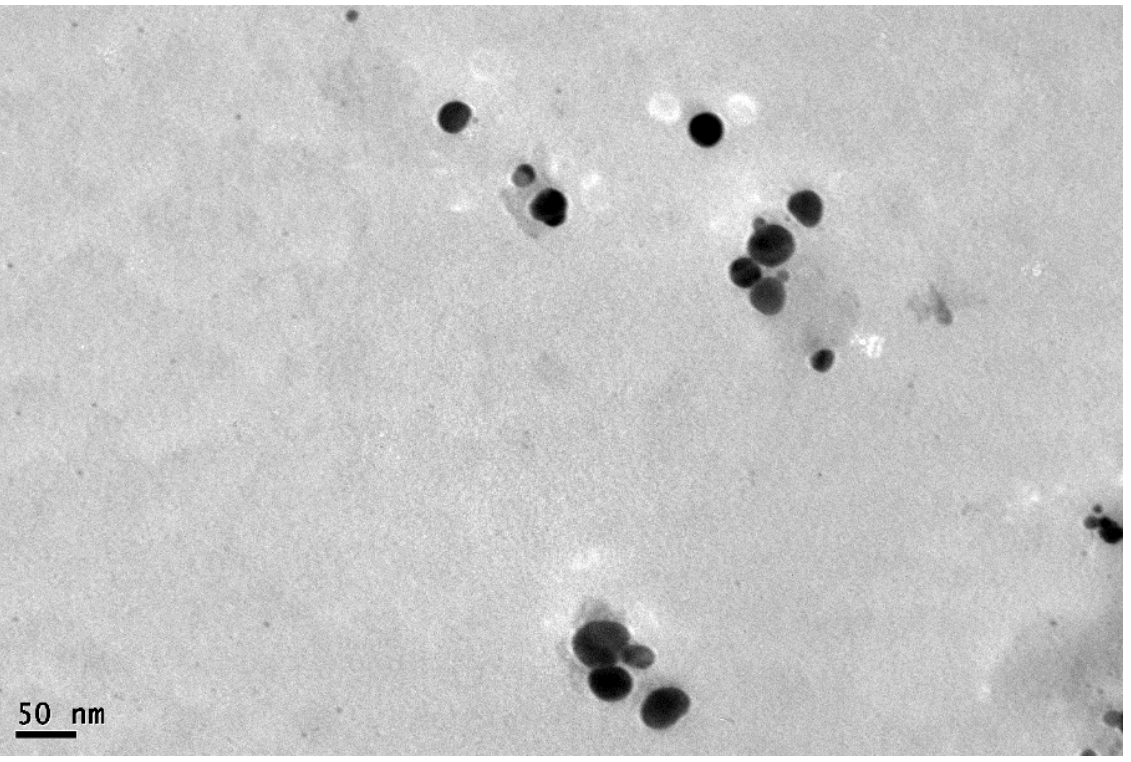

c)

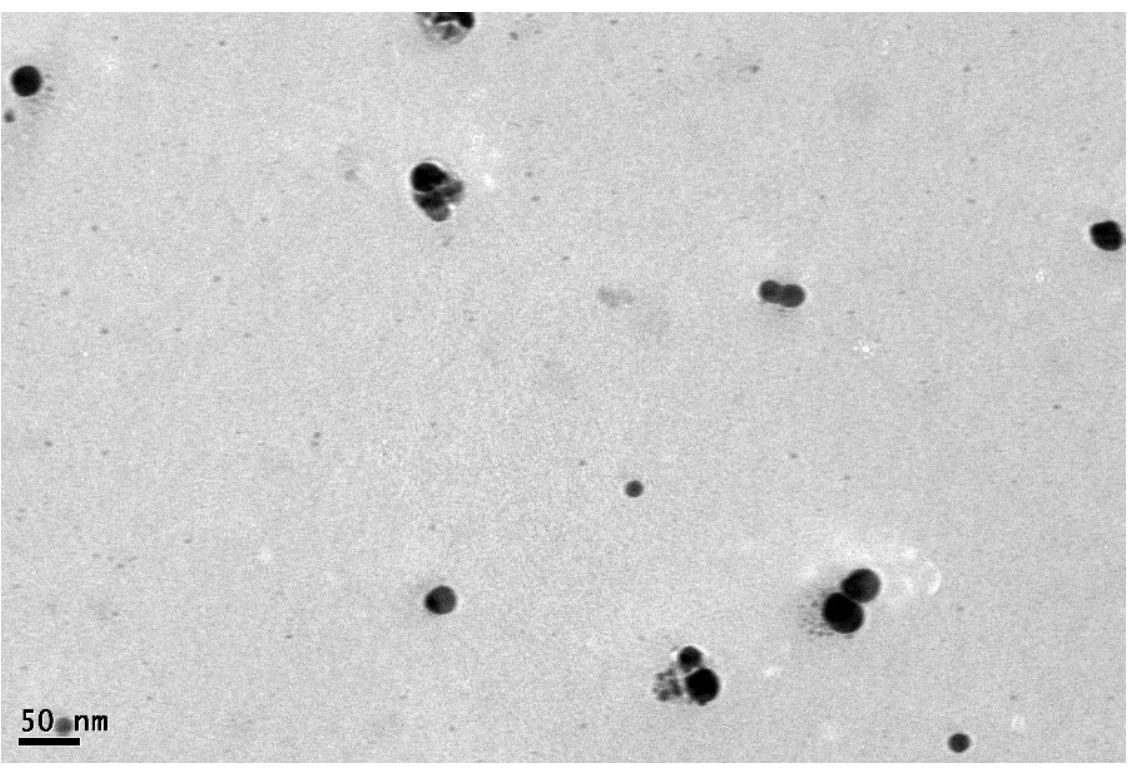

Figure S5. a) b) c), TEM images of silver nanoparticle synthesized in citrate rich phase acquired at different locations of the TEM grid 
a)

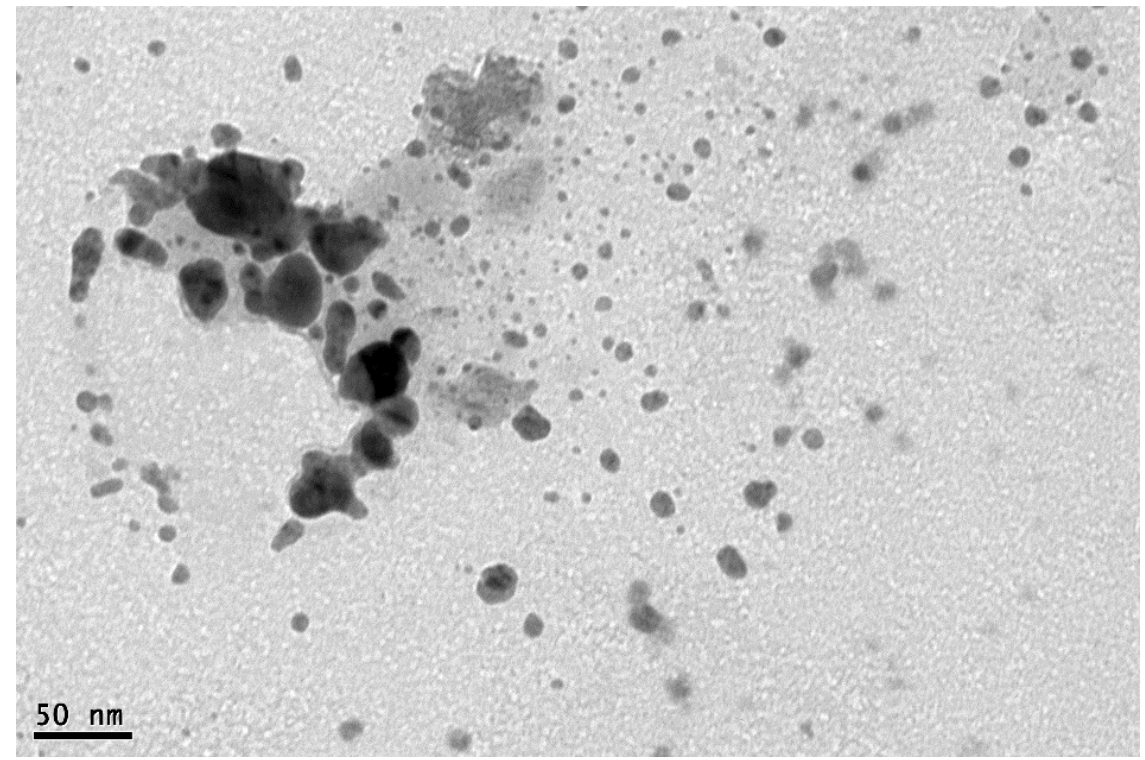

b)

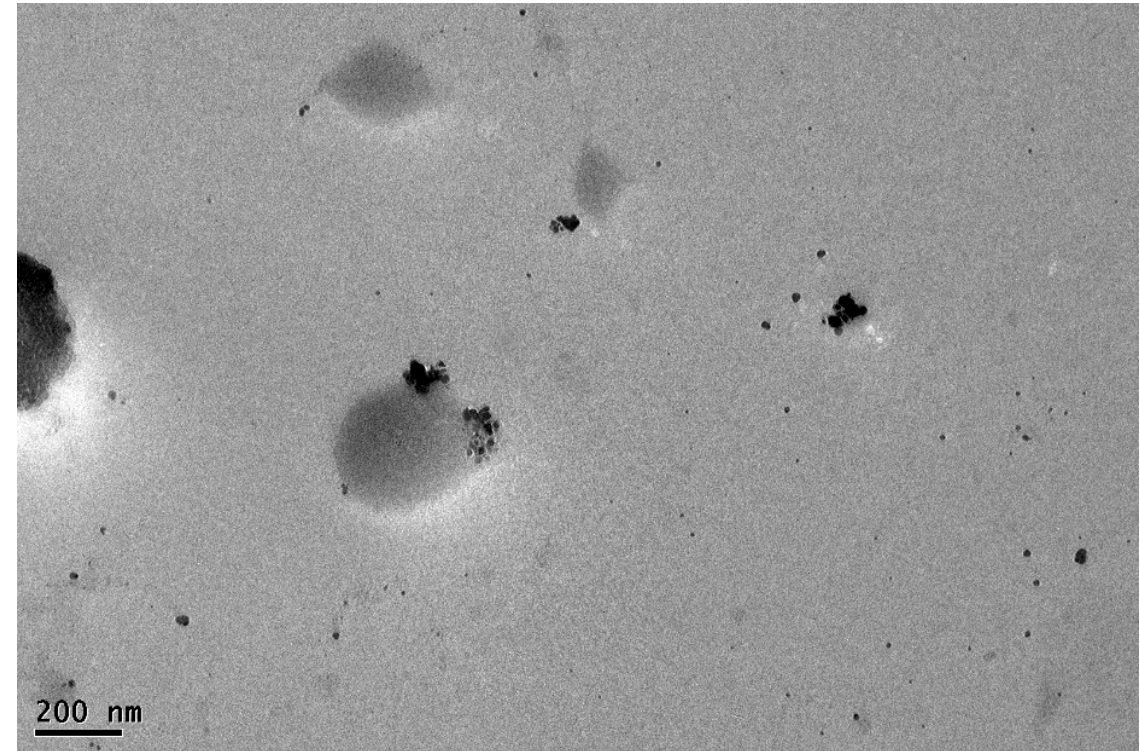

c)

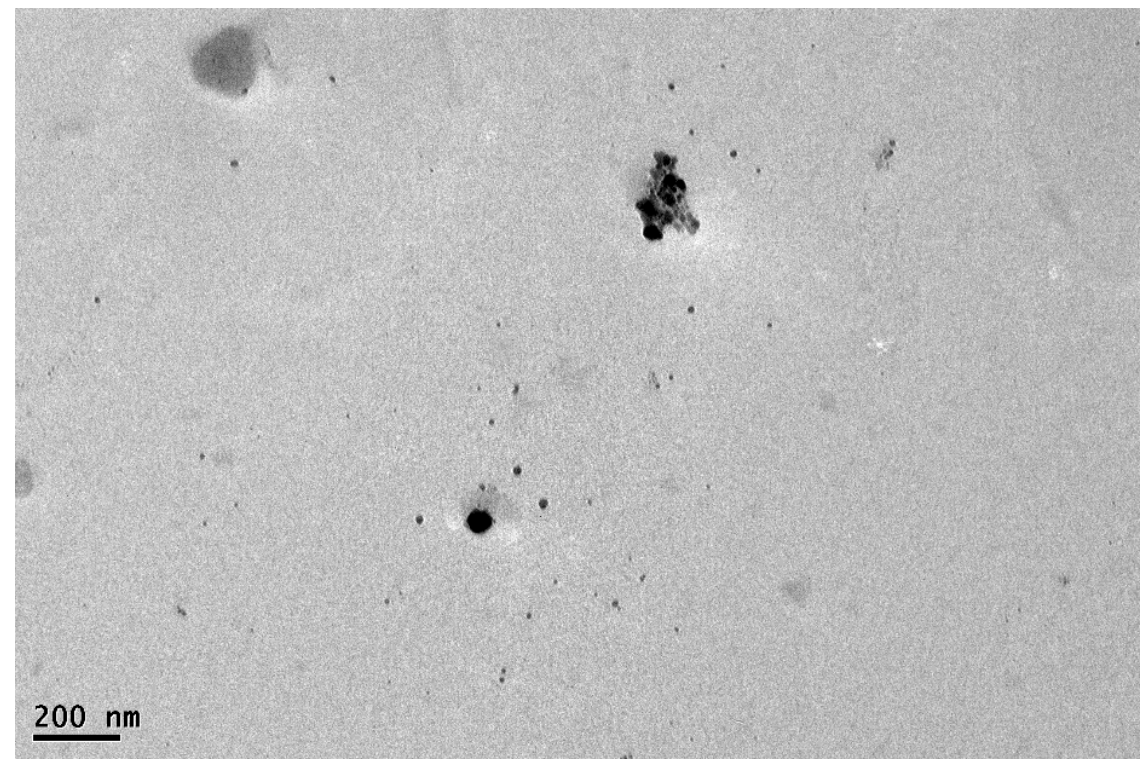

Figure S6. a) b) c), TEM images of silver nanoparticles synthesized in PEG rich phase acquired at different locations of the TEM grid. 


\section{S3. Trapping of nanoparticles at the two phase interface:}

Experiments were conducted to understand the transport of the synthesized nanoparticles in the aqueous two phase system (ATPS). For this, a drop (15 $\mu 1)$ of pure citrate solution (24 weight percent) was added on a glass slide. Later, $10 \mu \mathrm{l}$ of PEG solution ( 24 weight percent) containing silver nanoparticles and fluorescein dye was added on the top of citrate. This solution was observed under a microscope (Nikon NIS-E) at 20x. The video of the experiment is provided in the Supporting Movie M5.

It is observed that when the PEG solution is added to the citrate phase, an instability arises in the system. This is caused by the mass transfer between the two phases. Citrate transfers into the PEG phase and PEG into the citrate phase. As time proceeds, small droplets of citrate are observed in the PEG droplet. These citrate droplets merge to form larger droplets inside the PEG droplet. These droplets carry aggregates of nanoparticles at the interface. As the citrate droplets grow in size, they move downwards in the PEG droplet and merge into the continuous citrate phase. During the merging of citrate droplets in the continuous citrate phase, the aggregates of the nanoparticles are transported towards the interface of PEG droplet.

\section{S4. Droplet Coalescence:}

This experiment was conducted to study the coalescence of the droplets in ATPS. $15 \mu 1$ of pure citrate solution ( 24 weight percent) was mixed with $10 \mu 1$ of PEG solution (24 weight percent) containing nanoparticles and fluorescein dye. The droplet was pipetted in and out several times to ensure mixing. Later, the mixture was placed on a microscopic slide for observation. The solution was observed in a microscope at 20x. The PEG droplets were observed to be unstable and merge to form larger droplets. The nanoparticles in the PEG phase do not stabilize the droplets. Eventually, the PEG droplets merge and form one large PEG droplet phase. Since the time scale for droplets to completely merge to form one single drop is large, the video provided in the Supporting Movie M6 shows partial droplet coalescence.

\section{S5. Synthesis in pure citrate solution:}

To understand the effect of $\mathrm{NaOH}$ in the reduction process, the synthesis of silver nanoparticles was carried out at three different values of $\mathrm{pH} 4,7$ and 11 . Typically, $0.01 \mathrm{M}$ silver nitrate was added in $50 \mathrm{ml}$ of $25 \mathrm{wt} \%$ citrate solution. The $\mathrm{pH}$ was maintained by the addition of $\mathrm{NaOH}$ and $\mathrm{HNO}_{3}$. The motivation for choosing $\mathrm{HNO}_{3}$ was to prevent displacement reaction which would be possibly caused by the other acids. The solution with $\mathrm{pH} 4$ turned milky white while there was no color change atpH7. Nanoparticles were formed in citrate solution with $\mathrm{pH}$ 11. This was confirmed by the color change and the peak in UV-Vis absorption spectra. Figure S7 depicts the images of the solution and the corresponding UV-vis absorption spectra. 

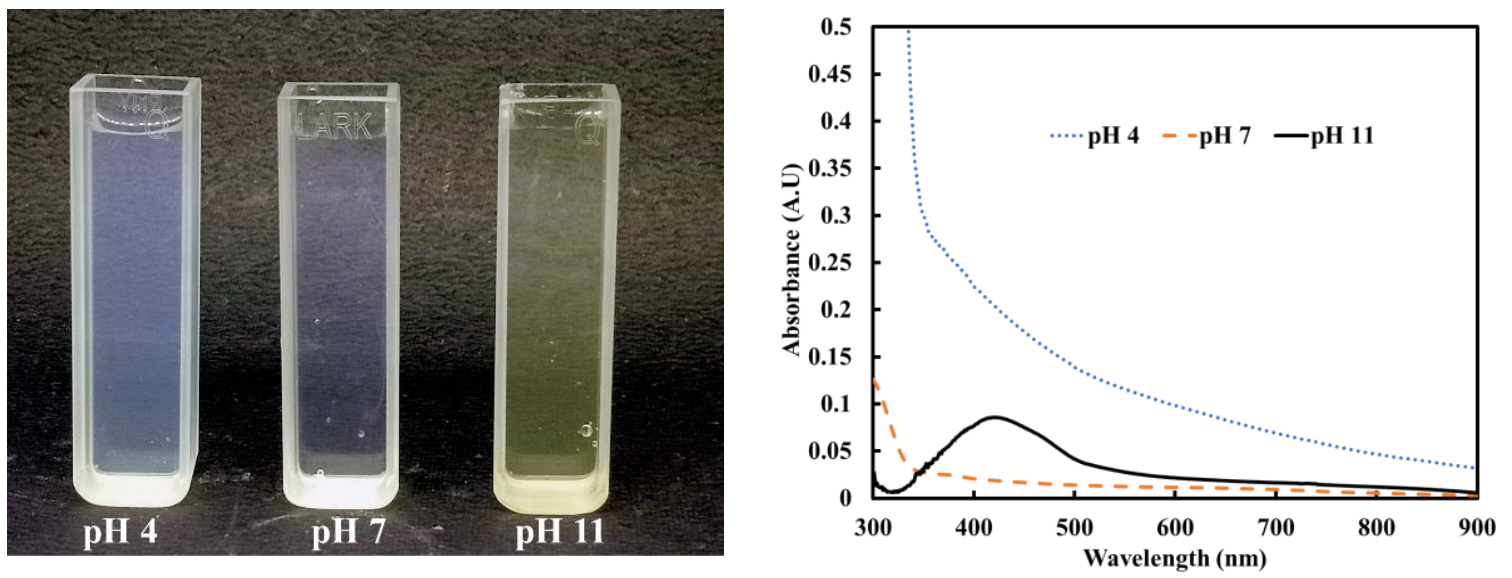

Figure S7. Effect of pH on synthesis of silver nanoparticles in citrate phase

Further, studies were performed to understand the effect of citrate concentration (in weight percent) on nanoparticle synthesis. For this, the synthesis of nanoparticles was carried out in different citrate weight percent solutions viz. 1,5,10, and15. The nanoparticles were synthesized at room temperature by the addition of $1 \mathrm{ml}$ of $0.01 \mathrm{M}$ silver nitrate to different concentrations of $50 \mathrm{ml}$ citrate solutions. Later, $\mathrm{NaOH}$ was added dropwise to all the solutions such that the $\mathrm{pH}$ of the solution is maintained at eleven. Further, $1 \mathrm{ml}$ of $0.01 \mathrm{M}$ silver nitrate was added dropwise to the citrate solution. The addition of $\mathrm{NaOH}$ to the solution enhanced the reduction potential of citrate ions allowing room-temperature synthesis of silver nanoparticles. The silver nanoparticles formed via the addition of $\mathrm{NaOH}$ were unstable at 1 weight percent as it turned black after some time. Figure $\mathbf{S 8}$ shows nanoparticles synthesized in the solution for different weight percent of citrate concentration in the presence of $\mathrm{NaOH}$.

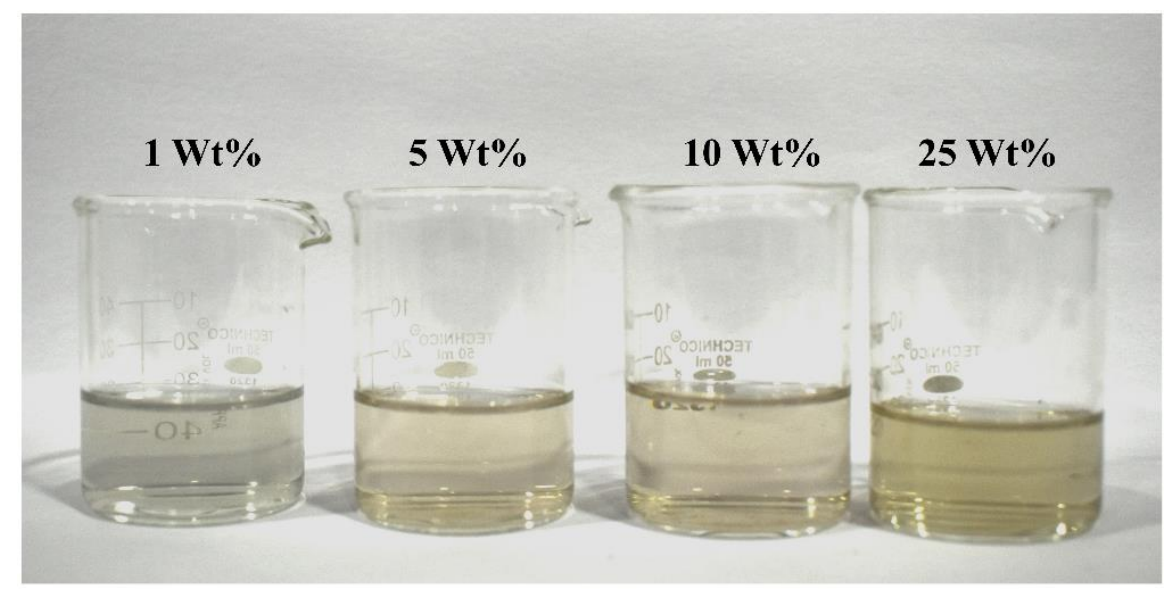

Figure S8. Silver nanoparticles synthesised in presence of $\mathrm{NaOH}$ for different concentrations of citrate solution.

We now describe the mechanism for the silver nanoparticle synthesis in the citrate phase. During the synthesis process, citrate $\left(\mathrm{C}_{6} \mathrm{H}_{5} \mathrm{O}_{7} \mathrm{Na}_{3}\right)$ reduces $\mathrm{Ag}^{+}$ions to metallic $\mathrm{Ag}$ atoms through the following reaction: ${ }^{2}$

$$
4 \mathrm{Ag}^{+}+\mathrm{C}_{6} \mathrm{H}_{5} \mathrm{O}_{7} \mathrm{Na}_{3}+2 \mathrm{H}_{2} \mathrm{O} \rightarrow 4 \mathrm{Ag}+\mathrm{C}_{6} \mathrm{H}_{5} \mathrm{O}_{7} \mathrm{H}_{3}+3 \mathrm{Na}^{+}+\mathrm{H}^{+}+\mathrm{O}
$$


The Ag atoms formed act as nucleation centers and catalyze the reduction of the remaining metal ions present in the solution. The formation of $\mathrm{Ag}$ nanoparticles was inferred by a change in solution color from colorless to a stable yellow tinge. For low citrate concentrations (less than $100 \mathrm{mM}$ ), nanoparticle formation did not require the addition of $\mathrm{NaOH}$. At low concentrations of citrate addition of $\mathrm{NaOH}$ enhanced the reduction and it was possible to synthesize nanoparticles at room temperature. The reaction mechanism for this case is illustrated in Figure S9a. At a low concentration of citrate, the $\mathrm{Ag}^{+}$ions form a complex with citrate by binding to the adjacent $\mathrm{C}=\mathrm{O}$ and $\mathrm{C}-\mathrm{O}$ bonds. This is followed by a reduction to silver atoms and nucleation within the medium.

At higher citrate concentrations (above $150 \mathrm{mM}$ ), large lumps of aggregated silver particles were formed when $\mathrm{NaOH}$ was not added, giving the solution a dark grey tinge. This is attributed to the destabilization effects at the high ionic strength of the solution causing aggregation of nanoparticles. ${ }^{3}$ To mitigate this effect, $\mathrm{NaOH}$ was added at higher citrate concentrations. Addition of $\mathrm{NaOH}$ enhanced the reducing power of citrate, through a threestep mechanism described in Figure S9b: First $\mathrm{Ag}_{2} \mathrm{O}$ is formed in the solution. The amount formed is dependent upon the ratio of nitrate to $\mathrm{NaOH}$. In the second step, the silver oxide redissolves within the solution, creating an $\mathrm{Ag}^{+}$complex. In the third step, the $\mathrm{Ag}^{+}$complex reduces and nucleates within the medium, which is accelerated by increasing the $\mathrm{NaOH}$ concentration. Thus, the addition of $\mathrm{NaOH}$ helps in the room temperature synthesis of silver nanoparticles at high concentrations of citrate.

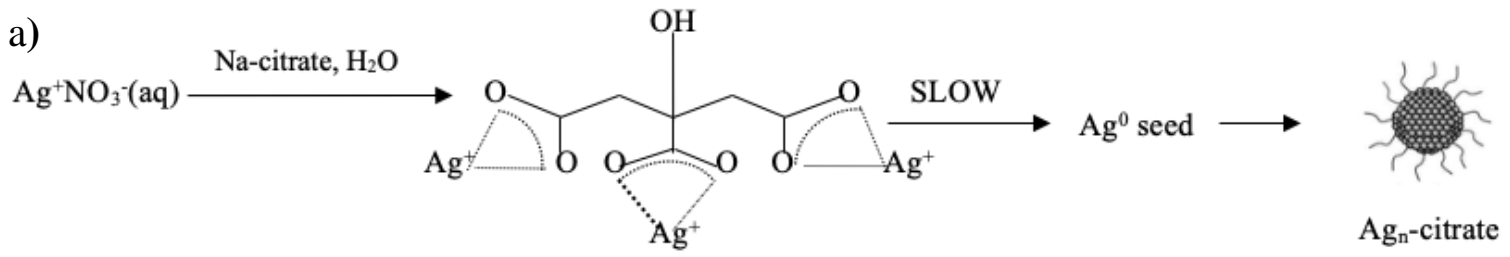

a)

b)

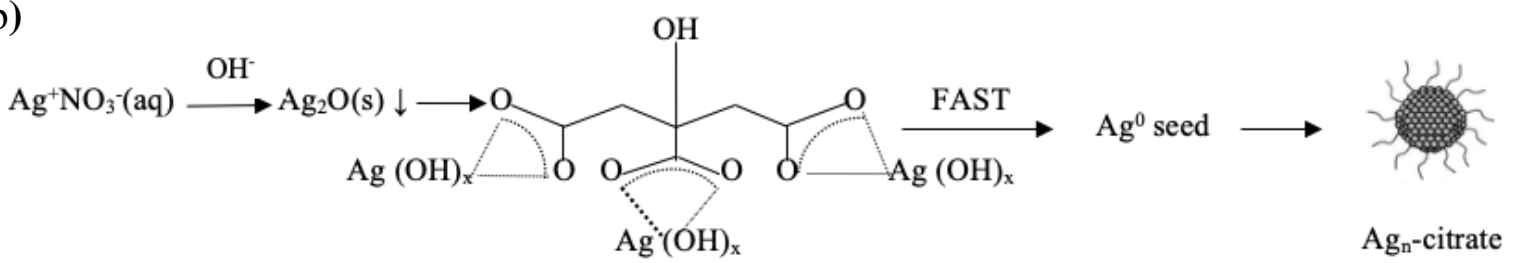

b)

Figure S9. Reduction mechanism of silver ions with tri-sodium citrate as a reducing agent a) without $\mathrm{NaOH}$ b) with $\mathrm{NaOH}$. 


\section{S6. Synthesis in pure PEG solution:}

To understand the effect of $\mathrm{pH}$ in the synthesis of nanoparticles in PEG phase. Experiments were performed for the synthesis of silver nanoparticles at three different values of $\mathrm{pH}(4,7,11)$. The $\mathrm{pH}$ pf PEG solutions was initially brought to 4,7,11 by the addition of $\mathrm{NaOH}$ and $\mathrm{HNO}_{3}$. Later, $1 \mathrm{ml}$ of $0.01 \mathrm{M}$ silver nitrate was added to the solution. It was observed that nanoparticles were formed only in PEG solution with $\mathrm{pH} 11$ (Figure S10). Whereas solutions with $\mathrm{pH} 4$ and 7 did not undergo any changes. The UV-Vis absorption spectra confirmed the formation of silver nanoparticles at $\mathrm{pH}$ 11(Figure S10).
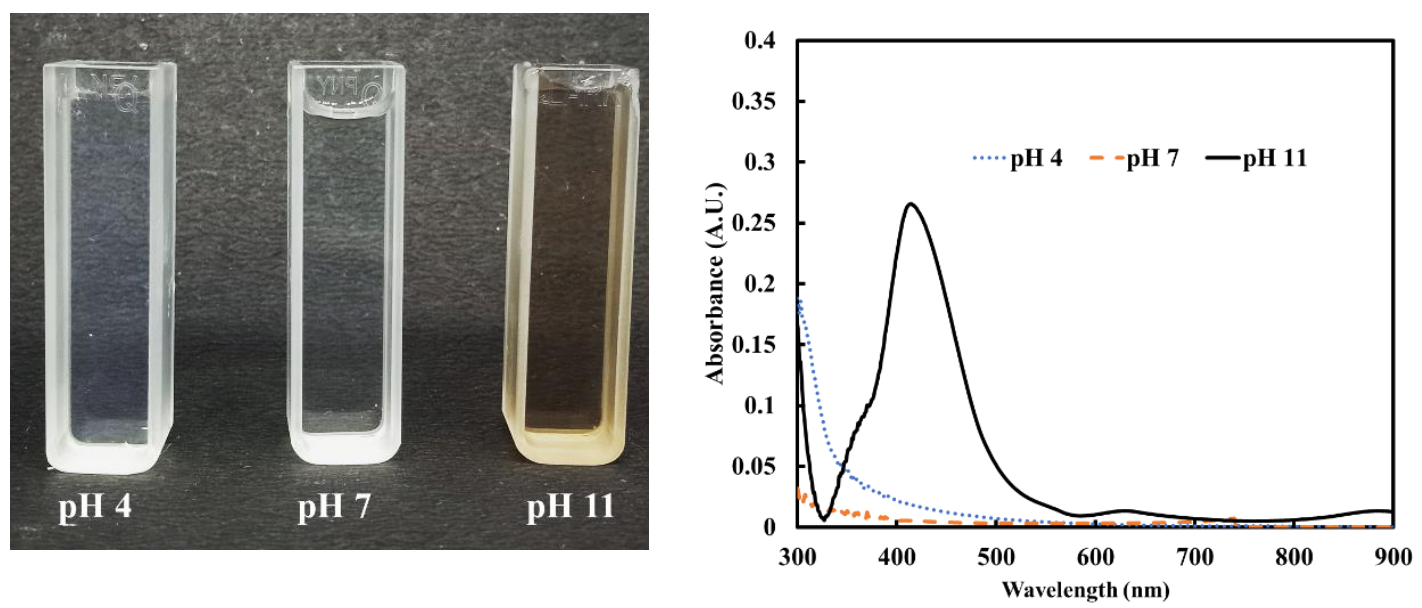

Figure S10. Effect of $\mathrm{pH}$ on synthesis of silver nanoparticles in PEG phase.

Further, experiments were conducted to study the effect of PEG concentration. Initially, $1 \mathrm{M}$ $\mathrm{NaOH}$ was added to $50 \mathrm{ml}$ PEG solutions with different concentrations. The amount of $\mathrm{NaOH}$ is added such that the $\mathrm{pH}$ is maintained at 11 . Further, $0.5 \mathrm{ml}$ of $0.01 \mathrm{M}$ silver nitrate was added to the solution as a precursor. It was observed that the solution turned yellow to brown based on the concentration of PEG (Figure S11).

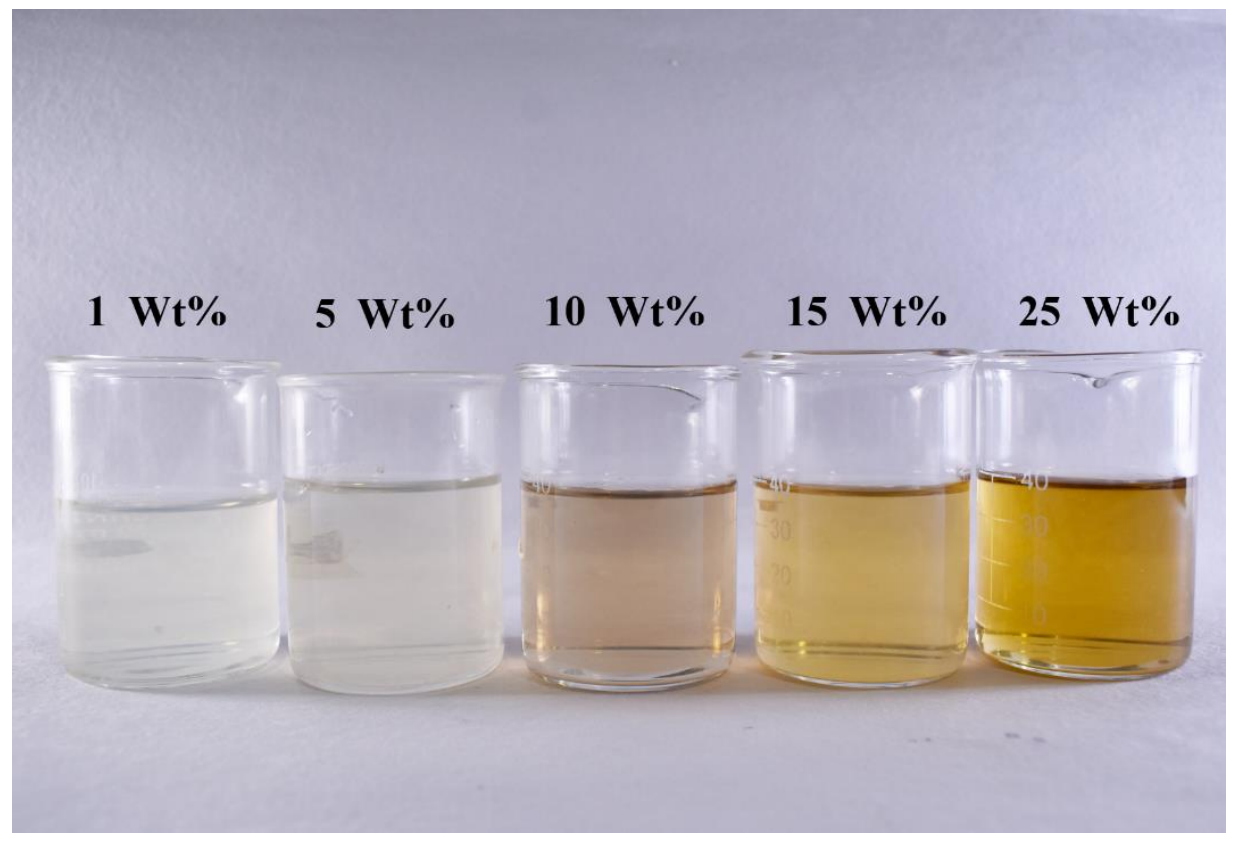

Figure S11. Synthesis of silver nanoparticles in different concentration of PEG solutions. 
In several previous studies dealing with longer chain variants of polyethylene glycol (PEG), the reaction time for successful reduction into stable nanoparticles varied from 1-3 hours at temperatures as high as $100^{\circ} \mathrm{C}-200^{\circ} \mathrm{C} .{ }^{4-6}$ This is due to the weak reducing power of PEG. However, the use of $\mathrm{NaOH}$ as a catalyst for the reduction reaction enabled us to successfully synthesize stable nanoparticles using PEG 6000 at room temperature with a reaction time of only 15-20 minutes. The reaction mechanism for this is depicted in Figure S12. Due to the presence of $\mathrm{NaOH}$ in $\mathrm{PEG}$, the hydrogen atom in the hydroxyl group in PEG is dehydrogenized by $\mathrm{OH}^{-}$ions derived from sodium hydroxide to form PEG alkoxide, which acts as the reducing ion for $\mathrm{Ag}^{+}$ions. ${ }^{7}$ The formation of PEG alkoxide in basic solutions increases the reducing power of PEG and enhances the reaction rate.

$$
\begin{gathered}
\mathrm{PEG}+\mathrm{Ag}^{+} \quad \rightarrow \quad[\mathrm{Ag}-\mathrm{PEG}]^{+} \\
{[\mathrm{Ag}-\mathrm{PEG}]^{+} \underset{\text { Stirring }}{\rightarrow}[\mathrm{Ag}-\mathrm{PEG}] \downarrow}
\end{gathered}
$$

a)

$$
\begin{aligned}
& \mathrm{Na}-\mathrm{OH} \rightarrow \mathrm{Na}^{+}+\mathrm{OH}^{-} \\
& \mathrm{R}-\mathrm{OH} \rightarrow \mathrm{R}^{-} \mathrm{O}^{-}+\mathrm{H}^{+} \\
& \mathrm{R}-\mathrm{OH}+\mathrm{OH}^{-} \rightarrow \mathrm{R}^{-} \mathrm{COO}^{-}+\mathrm{H}_{2} \uparrow \\
& \mathrm{R}-\mathrm{COO}^{-}+\mathrm{Ag}^{+} \rightarrow[\mathrm{RCOO}-\mathrm{Ag}] \downarrow
\end{aligned}
$$

b)

Figure S12. Reaction mechanism for the nanoparticle synthesis using PEG as a reducing agent a) without $\mathrm{NaOH}$ b) with $\mathrm{NaOH}$

\section{S7. Large Scale Synthesis of silver nanoparticles}

A major advantage of the proposed process is its scalability to large-scale production of silver nanoparticles. To demonstrate this, the synthesis of silver nanoparticles was carried out on a one litre scale. Initially, $500 \mathrm{ml}$ of $24 \mathrm{wt}$ \% PEG solution was prepared. The initial pH of the solution was brought to 11 . Further, $10 \mathrm{ml}$ of $0.01 \mathrm{M}$ silver nitrate was added to this solution dropwise. The instantaneous color change of the solution to yellow indicated the formation of silver nanoparticles. The UV-Vis absorption spectra (Figure S13) confirmed the formation of silver nanoparticles. Later, $500 \mathrm{ml}$ of $24 \mathrm{wt}$ \% citrate solution was added to the solution and the overall system was stirred at $600 \mathrm{rpm}$ for one minute. The solution was transferred to a separating funnel where the system was allowed to phase separate. The two phases consisted of PEG rich phase and citrate rich phase with nanoparticles trapped at the interface. Both the phases and nanoparticles at the interface were separated using a separating funnel. The separated nanoparticles were washed and re-dispersed in deionized water. UV-Vis analysis was performed for the re-dispersed nanoparticles to confirm if the optical properties of the nanoparticles are retained after separation. 
Later, the remnant solution was recycled and reused for the synthesis of silver nanoparticles. Here, the nanoparticles were synthesized in PEG rich phase. For this, the initial $\mathrm{pH}$ was set to 11 and $10 \mathrm{ml}$ of $0.01 \mathrm{M}$ silver nitrate solution was added dropwise. A color change confirmed the formation of silver nanoparticles to yellow and the absorption spectra indicated the formation of silver nanoparticles. It was observed that the peak was broader and with less absorption intensity than that seen in the first run. This indicated a loss in reducing the power of PEG with each run. The citrate rich phase was added to the PEG rich phase and the solution was stirred. Later, the nanoparticles and the phases were separated. The phases were again available for reuse in synthesis.

The UV-Vis results for each run is described in Figure S13. It shows that the quantity of particles synthesized decreased with each run. Also, the width of LSPR peaks increased with each run indicating an increase in size distribution. This confirms that the solutions can be recycled and reused for particle synthesis.
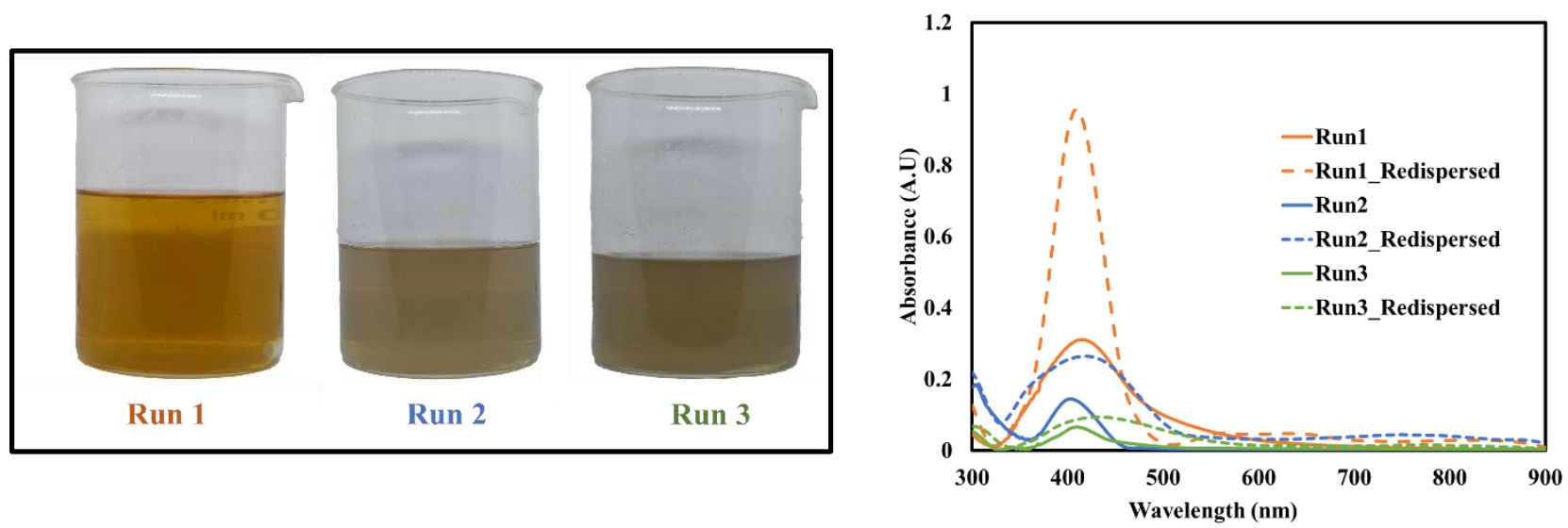

Figure S13. Large scale synthesis of silver nanoparticles and recycling of PEG and citrate phases.

\section{S8. Use of Ascorbic acid for the synthesis of nanoparticles in citrate phase}

Spherical shaped nanoparticles were synthesized using ascorbic acid as a co-reducing agent in PEG and citrate phase.

Here, $1 \mathrm{ml} 0.1 \mathrm{M}$ Ascorbic acid was added to either of the phases before addition of silver nitrate for the synthesis. After this addition, the PEG/citrate phase turned yellow indicating the formation of silver nanoparticles. The UV-Vis absorption spectrum exhibited a peak at $410 \mathrm{~nm}$ (Figure S14) confirming this.

Later, the particles were separated via interfacial trapping and washed for particle morphology characterization. The TEM image in Figure S15 indicates the nanoparticles formed are monodisperse. Particles with size $7 \mathrm{~nm}$ and $9 \mathrm{~nm}$ were formed in citrate and PEG phase respectively.

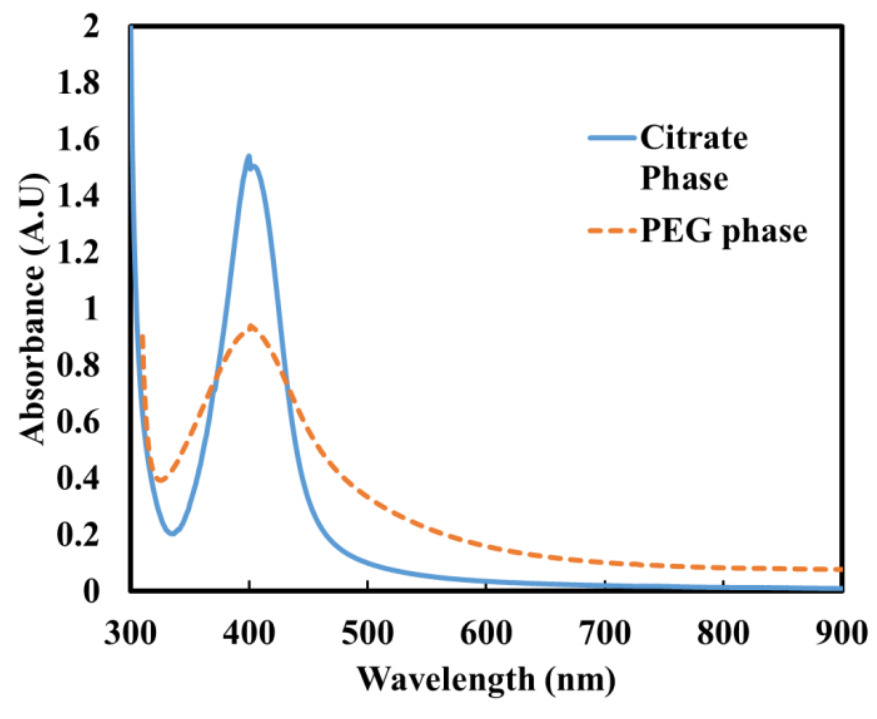


Figure S14. UV-Vis absorption curve for the nanoparticles synthesized with ascorbic acid as co-reducing agent in PEG and citrate phases.
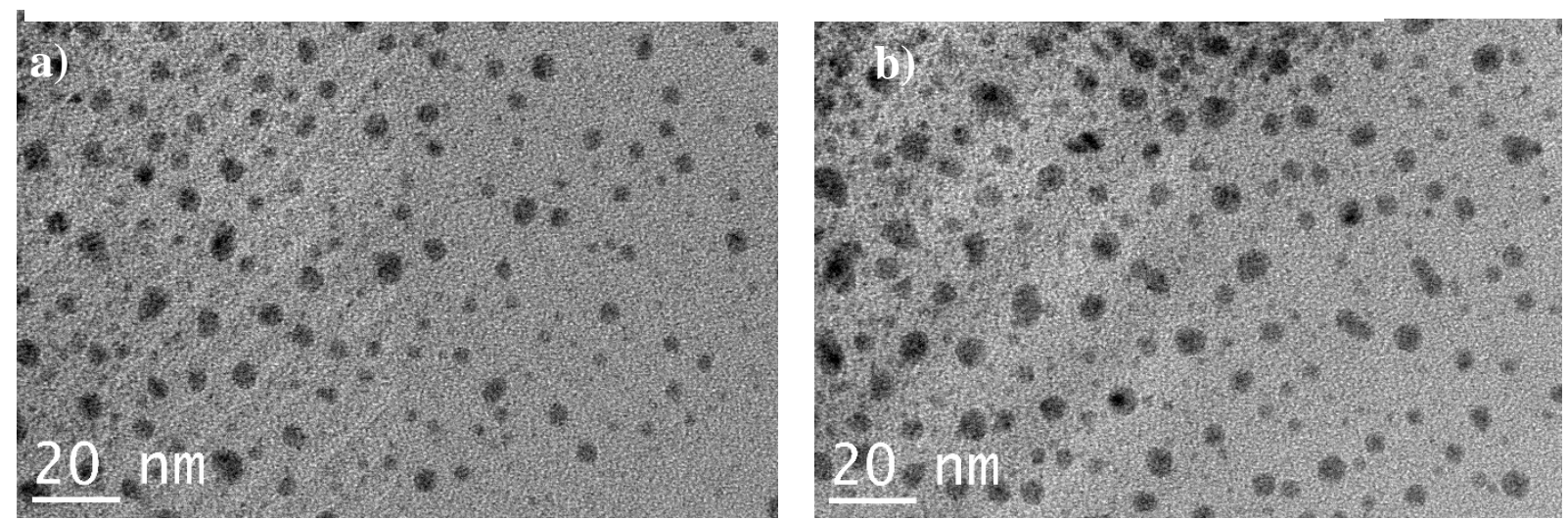

Figure S15. TEM image of silver nanoparticles synthesized using ascorbic acid in a) the citrate phase b) PEG phase indicating monodispersed particles.

\section{S8. Synthesizing cube shaped nanoparticle in PEG phase}

Cube shaped nanoparticles were synthesized using Polyvinyl pyrrolidine (PVP) as a template for nanoparticle synthesis. The synthesis was carried out in PEG phase.

Initially, $1 \mathrm{ml}$ of $0.1 \mathrm{M}$ PVP solution was added to PEG phase prior to addition of silver nanoparticles. Later, $1 \mathrm{ml}$ of $0.01 \mathrm{M}$ silver nitrate was added to the PEG phase. The solution turned brown indicating that nanoparticles were formed. The formation of a hump at $418 \mathrm{~nm}$ and a peak at $573 \mathrm{~nm}$ in UV-Vis absorption spectrum (Figure S16a) indicate the formation cube shaped nano particles. It is known that PVP controls the growth of nanoparticles and helps in obtaining a cube shaped morphology. Figure S16 b shows the SEM image for the cube shaped nanoparticle synthesized using PVP as a template.

a)

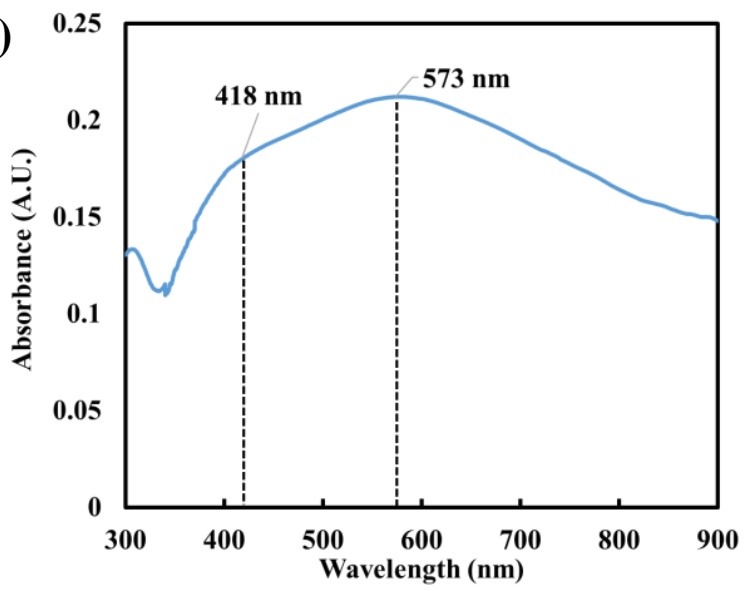

b)

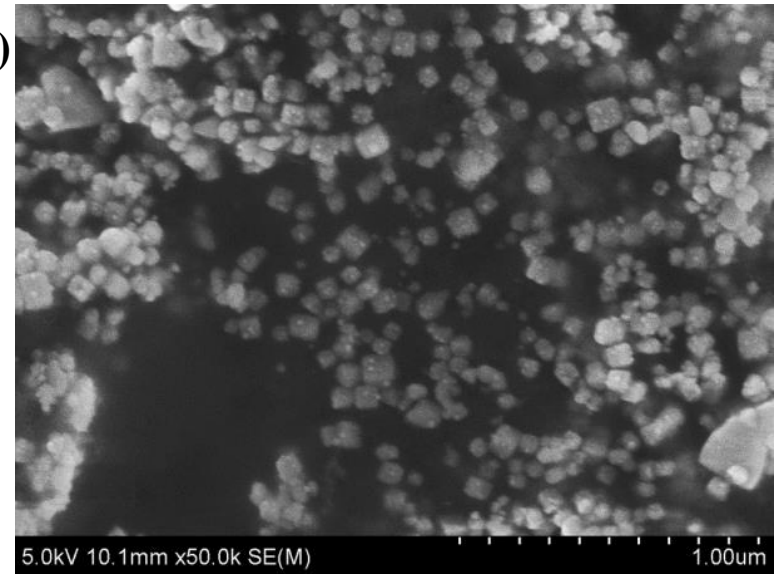

Figure S16.a) UV-Vis absorption spectra and b) SEM image indicating the formation of cube shaped nanoparticles using PVP as a template in the PEG phase. 


\section{S8. References}

(1) Hatti-Kaul, R. Aqueous Two-Phase Systems A General Overview. Mol. Biotechnol. 2001, 19, 269-277.

(2) Suriati, G.; Mariatti, M.; Azizan, A. Synthesis of Silver Nanoparticles by Chemical Reduction Method: Effect of Reducing Agent and Surfactant Concentration. Int. J. Automot. Mech. Eng. 2014, 10 (1), 1920-1927.

(3) Thanh, N. T. K.; Maclean, N.; Mahiddine, S. Mechanisms of Nucleation and Growth of Nanoparticles in Solution. Chem. Rev. 2014, 114 (15), 7610-7630.

(4) Popa, M.; Pradell, T.; Crespo, D.; Calderón-Moreno, J. M. Stable Silver Colloidal Dispersions Using Short Chain Polyethylene Glycol. Colloids Surfaces A Physicochem. Eng. Asp. 2007, 303 (3), 184-190.

(5) Mtimet, I.; Lecamp, L.; Kebir, N.; Burel, F.; Jouenne, T. Green Synthesis Process of a Polyurethane-Silver Nanocomposite Having Biocide Surfaces. Polym. J. 2012, 44 (12), 1230-1237.

(6) Fahmy, A.; El-Zomrawy, A.; Saeed, A. M.; Sayed, A. Z.; El-Arab, M. A. E.; Shehata, H. A.; Friedrich, J. One-Step Synthesis of Silver Nanoparticles Embedded with Polyethylene Glycol as Thin Films. J. Adhes. Sci. Technol. 2017, 31 (13), 1422-1440.

(7) Xiao, Y.; Jiang, J.; Huang, H. Chemical Dechlorination of Hexachlorobenzene with Polyethylene Glycol and Hydroxide: Dominant Effect of Temperature and Ionic Potential. Sci. Rep. 2014, 4, 1-6. 